\author{
K. Hadjadj, M. Benaissa, M. Mahammedi, G. Belkacem \& L. Guerine
}

\title{
Les peuplements à Fraxinus dimorpha (Oleaceae) dans les Monts des Ksour occidentaux (Sud ouest algérien): diversité phytocenotique, dynamique structurale et perspectives de conservation
}

\begin{abstract}
Hadjadj, K., Benaissa, M., Mahammedi, M., Belkacem, G. \& Guerine, L.: Les peuplements à Fraxinus dimorpha (Oleaceae) dans les Monts des Ksour occidentaux (Sud ouest algérien): diversité phytocenotique, dynamique structurale et perspectives de conservation. - Fl. Medit. 30: 155-165. 2020. — ISSN: 1120-4052 printed, 2240-4538 online.

The stands of Fraxinus dimorpha (Oleaceae) in the Western Ksour Mountains (South West Algeria): phytocenotic diversity, structural dynamics and conservation prospectives. - Fraxinus dimorpha is a threatened species appearing on the IUCN red list. It structures a valuable preforest heritage in the Western Ksour Mountains (Algeria), both for its ecological and social value. Thus, the aim of this first study is to contribute to the knowledge of the floristic diversity and the structural dynamics of Fraxinus dimorpha stands in this area.

The floristic diversity's analysis of Fraxinus dimorpha preforests in western Ksour Mountains has revealed the existence of 188 specific and subspecific taxa belonging to 40 families and 145 genera. The biogeographic spectrum indicates the dominance of Mediterranean taxa with 95 species. 30 endemic species have been identified of which 19 are Algerian-Moroccan ones. The Analysis of biological types indicated the dominance of therophytes over the other life forms (69 taxa, or $36.70 \%$ ). The study of structural dynamics (diametric classes) showed the dominance of perches (42\%), little wood (37\%) and medium wood $(21 \%)$.
\end{abstract}

Key words: Floristic diversity, ash tree, Algeria, North Africa.

\section{Introduction}

Le frêne dimorphe (Fraxinus dimorpha Coss. \& Durieu) fait partie de la famille des Oléacées. C'est une essence de montagnes, endémique de l'Afrique du Nord avec une répartition restreinte au Maroc et en Algérie (Charco 2001; Fennane \& Ibn Tattou 2005; Fennane \& al. 2007; Euro+Med 2015). En Algérie le frêne dimorphe est localisé dans l'Aurès (notamment dans les forêts des Oued Ensigha, Oued Boulgem et Oued Tamza), Djebel Tougour près de la province de Batna, la vallée de l'Oued Abdi près de la province de Constantine, Bellezma, Djbel M'zi (Monts des Ksour), Toudja et les Monts de la Kabylie (Battandier \& Trabut 1895; Charco 2001). Au Maroc, il existe à l'état disséminé dans l'Haut Atlas (Ayachi, Maâsker, Vallée de l'Ourika près de Marrakech), l'Haut Atlas 
central principalement à Ait M'hamed, près de la province d'Azilal, entre 1300 et $1700 \mathrm{~m}$ d'altitude, Moyen Atlas (Nord Est du Moyen Atlas et Moyen Atlas central), l'Anti Atlas (Monts Saghro), Monts de Berkine et l'Atlas Saharien (Battandier \& Trabut 1895; Boudy 1950; Charco 2001; Fennane \& Ibn Tattou 2005; Fennane \& al. 2007; Taleb \& Fennane 2008; Euro+Med 2015).

L'espèce pousse dans les roches calcaires ou siliceuses moyennes Il préfère les habitats ouverts et ensoleillés, mais peut être trouvée dans les habitats ombragés. Il fleurit de Février à Mars et produit des fruits de Juillet à Septembre (Emberger 1938; Abdessemed 1981; Charco 2001; Genin \& al. 2016). C'est une essence xérophyte qui préfère les bioclimats arides et semi-arides, tempérés et frais avec une température entre -20 à $35^{\circ} \mathrm{C}$. L'espèce tolère également le climat très chaud et sec des montagnes bordant le Sahara (Emberger 1938; Genin \& al. 2016).

Fraxinus dimorpha est utilisé en médecine populaire pour ses effets diurétiques, laxatifs, antirhumatismaux et purgatifs doux ainsi que pour traiter la constipation, l'hydropisie, l'arthrite, la douleur rhumatismale, la cystite, la fracture osseuse et le cuir chevelu. Les fruits et les graines sont utilisés comme épices pour leurs qualités culinaires, médicinales et aphrodisiaques (Bellakhdar 1997; Kostova \& Iossifova 2007; M'Sou \& al. 2016).

Compte tenu de la rareté voir même l'absence des études écologiques et dendrométriques réalisées sur le frêne dimorphe dans les Monts des Ksour, cette recherche constitue une première contribution s'inscrivant dans le cadre de la connaissance, la préservation et la conservation de cette essence signalée endémique de l'Afrique du Nord par Fennane \& al. (2007) et Dobignard \& Chatelain. (2010-2013) et figurant sur la liste rouge de l'UICN des espèces menacées (Rankou \& al. 2017).

\section{Matériels et méthodes}

\section{Zone d'étude}

Situés au sud de la Meseta oranaise et au nord de la plateforme saharienne, les Monts des Ksour occupent la partie la plus occidentale de l'Atlas saharien (Fig.1). Il s'agit d'une chaîne intracontinentale, formée de terrains méso-cénozoïques plissés lors de l'orogenèse alpine (Aït Ouali 1991; Kacemi \& al. 2011). Les Monts des Ksour est une région assez élevée avec certains sommets dépassant les 2000 m (Fillali 2011). Ils sont constitués de chaînons allongés, d'orientation SW-NE, séparés des hautes plaines oranaises au nord par l'accident nord-atlasique et de la plate-forme saharienne au sud par l'accident sudatlasique. Ils se prolongent à l'ouest par le Haut Atlas marocain et à l'est par l'Atlas Saharien central (Djebel l'Amour) et oriental (Monts des Ouled Naîls) (Yelles \& al. 2001; Fillali 2011).

\section{Méthodes}

Dans les Monts des Ksour, le frêne dimorphe se localise principalement au niveau des versants à forte pente rocailleuse, généralement dépassant $25 \%$. Devant cette situation nous avons opté pour un échantillonnage aléatoire dirigé qui tient compte de la variabilité des peuplements de frêne dimorphe rencontré. La liste des espèces est établie à partir de 84 relevés floristiques réalisés sur une superficie de $100 \mathrm{~m}^{2}$. 


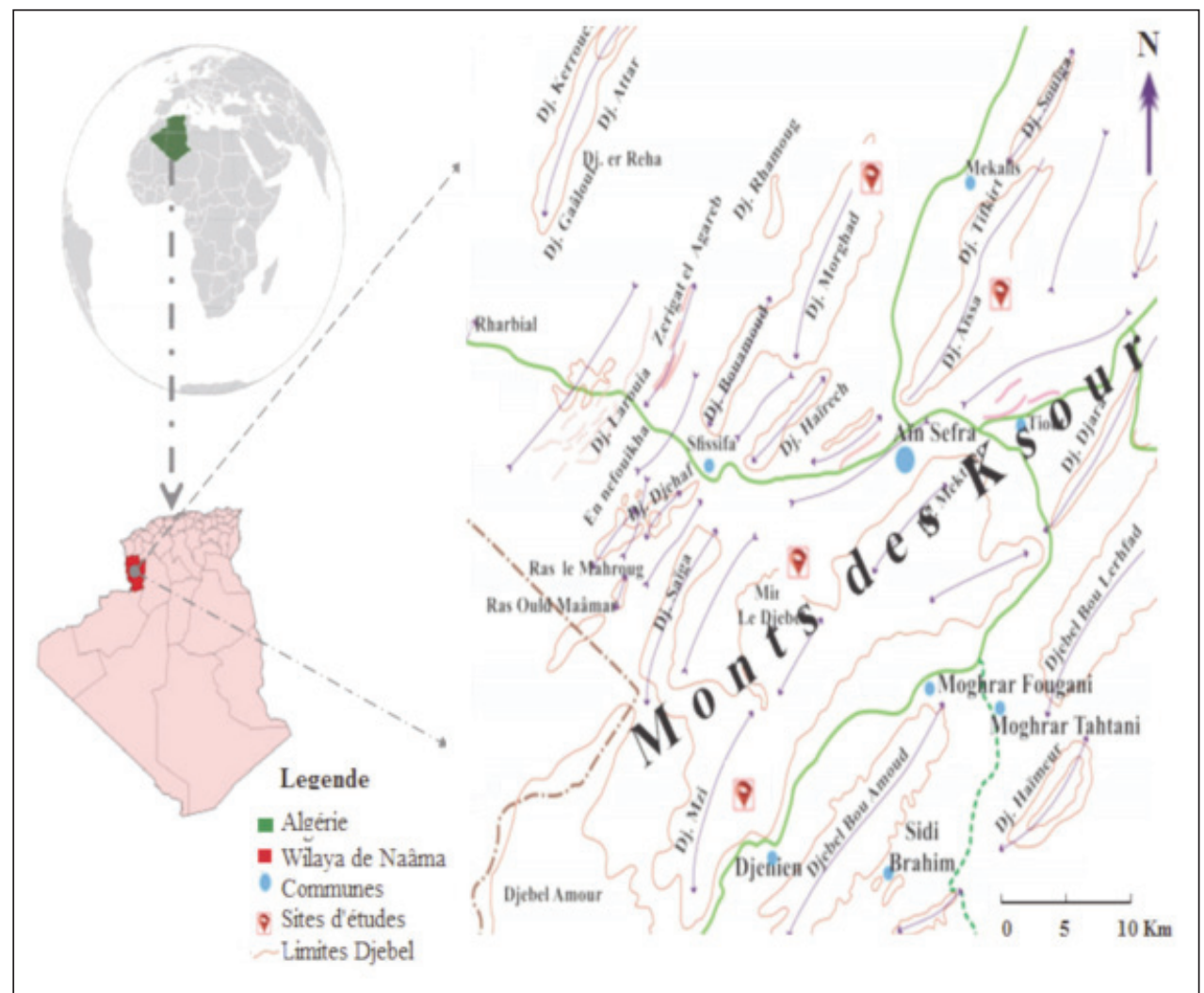

Fig. 1. Situation géographique de la zone d'étude.

Les espèces ont été identifiées selon la Nouvelle Flore d'Algérie et des régions désertiques méridionales (Quézel \& Santa 1962-1963), la Flore de l'Afrique du Nord (Maire 1952-1987), la Flore et végétation du Sahara (Ozenda 2004). La nomenclature a été actualisée pour les espèces recensées à la lumière des travaux récents compilés dans l'Index synonyme et bibliographique de la flore nord-africaine (Dobignard \& Châtelain 2010-2013).

Une stratification verticale de la flore inventoriée a été réalisée. Les différentes strates retenues dans cette proposition méthodologique sont celles décrites par Delassus (2015):

- Strate arborescente: constituée des arbres dont la hauteur est généralement $>7 \mathrm{~m}$;

- Strate arbustive supérieure: constituée d'arbustes hauts ou de jeunes arbres ayant des hauteurs comprises entre 3 et $7 \mathrm{~m}$;

- Strate arbustive basse: constituée d'arbustes bas ou de jeunes arbres de hauteur comprise entre 1 et $3 \mathrm{~m}$;

-Strate herbacée: constituée des espèces herbacées et des chaméphytes; jeunes plantules des espèces ligneuses, généralement inférieure à $1 \mathrm{~m}$.

Les espèces identifiées ont été indiquées par leur type biogéographique recomposé par Quézel \& Santa (1962-1963), Pignatti (1982), Dobignard \& Chatelain (2010-2013) et par 
leur type biologique. La classification à laquelle nous nous sommes référés est celle de Raunkiaer (1934). Elle se base sur la position qu'occupent les méristèmes en dormance par rapport au niveau du sol durant la saison difficile et se subdivise ainsi en: phanérophyte, chamaephyte, hémicryptophyte, thérophyte et géophytes.

En plus de l'étude du cortège floristique, une évaluation de la dynamique (structure verticale et structure diamétrique) des peuplements du frêne dimorphe a été réalisée. Pour ce faire nous avons mesuré la hauteur totale et la circonférence à $1,30 \mathrm{~m}$ du sol de tous les sujets rencontrés dans les relevés floristiques.

\section{Résultats et discussion}

Dans l'Atlas saharien occidental (Monts des Ksour), le frêne dimorphe a plutôt tendance à former des peuplements plus au moins ouverts entre $1800 \mathrm{~m}$ à $2200 \mathrm{~m}$ d'altitude. Il se mélange à la plupart des autres essences préforestières autochtones, il s'associe au chêne vert, pistachier térébinthe, pistachier de l'Atlas, genévrier de phénicie, genévrier oxycèdre.

Maire (1916) signale la présence du frêne dimorphe dans la plupart des montagnes sud oranaises de 1800 à 2100 m sur les grès et les calcaires. Il indique qu'il n'a pas rencontré l'espèce au niveau de Djebel Aissa.

Nos prospections sur terrain dans le territoire de Djebel Aissa ont démontré la présence de cette essence à partir de 1500 m jusqu'au 2100 m d'altitude notamment au niveau Ras Ettwil et oued ouled Benzziane.

\section{Diversité phytocenotique}

188 taxons appartenant à 40 familles et 145 genres ont été identifiés (Supplément Électronique 1), ce qui représente $4,37 \%$ de la flore totale algérienne estimée à 4300 espèces par Dobinard et Châtelain (2010-2013). Les familles les plus riches en taxons sont: Asteraceae avec 37 espèces (19,68\%), Poaceae 22 espèces (11,70 \%), Brassicaceae et Lamiaceae 14 espèces (7,44 \%), Caryophyllaceae 13 espèces (6,91 \%), Apiaceae 9 espèces $(4,78 \%$ ), Plantaginaceae et Fabaceae 8 espèces $(4,25 \%)$. Ces familles représentent $66,45 \%$ de la flore inventoriée.

Dans l'inventaire vertical de la végétation accompagnant le frêne dimorphe nous avons distingué les strates suivantes: arbustive supérieure, arbustive basse et herbacée.

Fraxinus dimorpha se trouve accompagné dans la strate arbustive supérieure par: Juniperus oxycedrus L., J. phoenicea L. subsp. phoenicea., Pistacia atlantica Desf., Quercus ilex subsp. ballota (Desf.) Samp., Phillyrea latifolia L., Acer monospessulanum L., Crataegus monogyna Jacq.

\section{Types géographiques}

L'étude biogéographique des végétaux est considérée par plusieurs auteurs (Paccalet 1981; Quézel 1983; Gaston 1991) comme un critère d'appréciation de la diversité biologique.L'analyse du spectre biogéographique des espèces recensées (Tableau 1) montre une dominance des espèces méditerranéennes (95 espèces) ce qui représente $50,53 \%$ de l'ensemble de la flore étudiée. Le type géographique de large répartition et cosmopolite 
Tableau 1. Types géographiques des espèces.

\begin{tabular}{|c|c|c|c|c|}
\hline Ensembles chorologiques & Nombre & $\%$ & Ensembles chorologiques & Nombre \\
\hline MEDITERRANEEN & 95 & \multirow{10}{*}{50,53} & Eurasiatique & 11 \\
\hline Méditerranéenne & 47 & & Paléotempéré & 6 \\
\hline Oroméditerranéenne & 3 & & Sud- Ouest européenne & 1 \\
\hline Ouest méditerranéenne & 16 & & Européenne & 5 \\
\hline Sud méditerranéenne & 2 & & $\begin{array}{l}\text { LARGE REPARTITION ET } \\
\text { COSMOPOLITES }\end{array}$ & $\mathbf{T}$ \\
\hline Circumméditerranéenne & 3 & & Cosmopolites & 2 \\
\hline Ibéromaurétanienne & 12 & & Méditerranéo-Atlantiques & 4 \\
\hline $\begin{array}{l}\text { Afrique nord, Espagne, } \\
\text { Caucasase, Turkey }\end{array}$ & 1 & & Euro-méditerranéennes & 15 \\
\hline Afrique du nord & 11 & & $\begin{array}{l}\text { Macaronésiennes- } \\
\text { méditerranéennes }\end{array}$ & 3 \\
\hline ENDEMIQUES & 30 & & $\begin{array}{l}\text { Macaronésiennes, } \\
\text { méditerranéennes, Asie, } \\
\text { occidental }\end{array}$ & 2 \\
\hline Nord-africaines & 5 & \multirow{7}{*}{15,96} & Paléotropicales & 2 \\
\hline Algéro-marocaines & 19 & & Méditerranéenne Asiatiques & 2 \\
\hline $\begin{array}{l}\text { Algéro-Marocain-Tunisie, } \\
\text { Canarie }\end{array}$ & 1 & & $\begin{array}{l}\text { Eurasiatique. Nord Africaine. } \\
\text { Tripoli }\end{array}$ & 3 \\
\hline $\begin{array}{l}\text { Endémiques ibéro- } \\
\text { marocain }\end{array}$ & 1 & & Canarie-Méditerranéen. & 1 \\
\hline $\begin{array}{l}\text { Algéro-Marocain, } \\
\text { Péninsule Ibérique }\end{array}$ & 4 & & $\begin{array}{l}\text { Méditerranéen-Irano- } \\
\text { Touranien }\end{array}$ & 3 \\
\hline SEPTENTRIONAL & 25 & & \multirow{2}{*}{$\begin{array}{l}\text { Méditerranéen, Saharo - } \\
\text { Sindien }\end{array}$} & \multirow{2}{*}{1} \\
\hline Circumboréale & 2 & & & \\
\hline
\end{tabular}

est représenté par 38 espèces $(21,20 \%)$, dont 15 taxons sont euro-méditerranéennes. Les espèces endémiques sont de l'ordre de 30 espèces $(15,96 \%)$, dont la majorité sont algéromarocaine (19 espèces). En fin, l'ensemble géographique septentrional compte 25 taxons $(13,30 \%)$. Les espèces eurasiatiques sont les plus abondantes avec 11 taxa.

\section{Types biologiques}

Les types ou formes biologiques qui désignent le comportement adaptatif de l'espèce, renseignent sur le type de la formation végétale, son origine et ses transformations. La répartition des types biologiques de l'ensemble de la flore de notre région d'étude est caractérisée par le schéma qui suit: $\mathrm{Th}>\mathrm{He}>\mathrm{Ch}>\mathrm{Nph}>\mathrm{Ph}>\mathrm{Géo}>\mathrm{Phl}$ (Fig. 2). Nous constatons une nette dominance des thérophytes qui constituent à elles seules un peu plus du tiers 


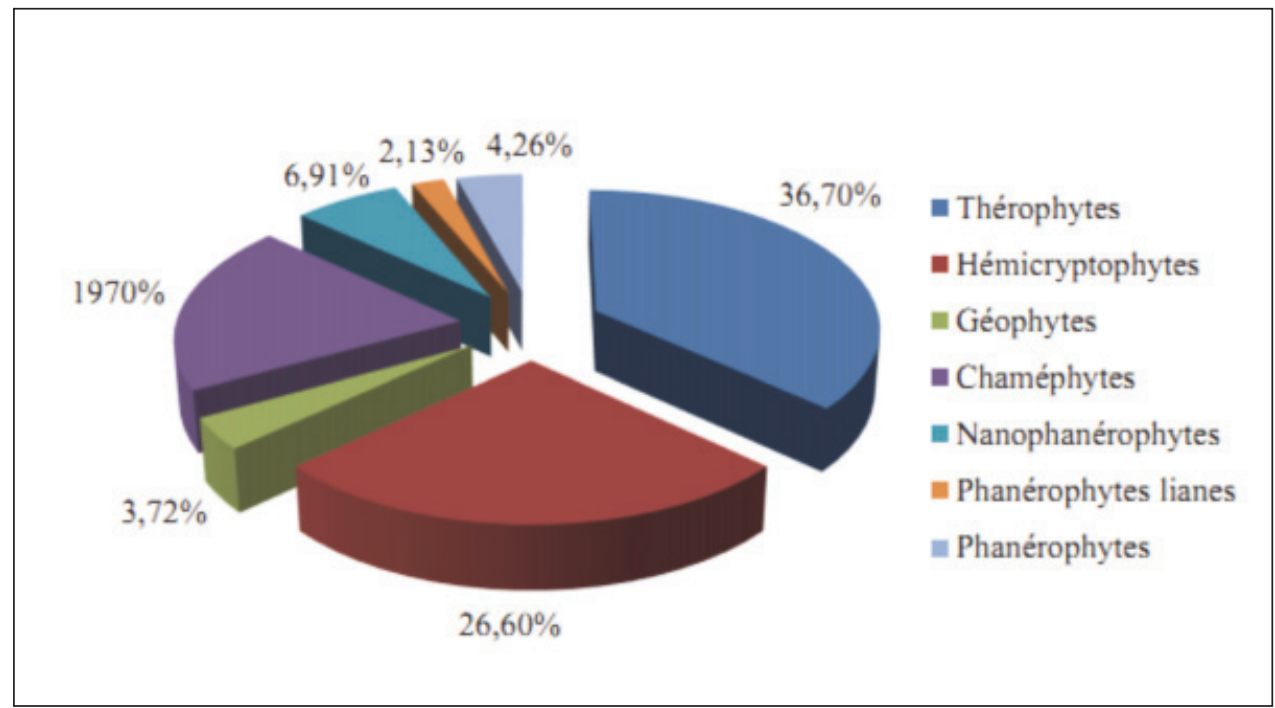

Fig. 2. Types biologiques.

de l'ensemble de la végétation, avec un total de 69 espèces, soit 36,70\%. En deuxième position viennent les hémicryptophytes et sont représentées par 50 taxons soit 26,60\%. Les chaméphytes occupent la troisième position avec 37 espèces ce qui représente 19,68 $\%$. Ces trois types biologiques (thérophytes, hémicryptophytes, chaméphytes) représentent une catégorie majoritaire par rapport aux autres types biologiques, leur taux cumulé est estimé à $82,98 \%$.

Le reste des espèces appartient aux nanophanérophytes $6,91 \%$, phanérophytes, 4,26\%, géophytes, 3,72\% et les phanérophytes lianes $2,13 \%$.

Cette richesse en thérophytes comme on le sait est une caractéristique des zones méditerranéennes et arides où domine un fort stress hydrique et une perturbation du milieu par le pâturage (Grime 1977; Daget 1980; Madon \& Médail 1996; Bonnet \& al. 1999; Barbero \& al. 2001; Hamada \& al. 2004; Aidoud 2005; Amghar \& al. 2008). En effet, la richesse en thérophytes représente un signe d'aridité du milieu et le phénomène de thérophytie est considéré par Daget (1980) comme une stratégie d'esquive des périodes défavorables.

\section{Structure des peuplements}

Le frêne dimorphe se présente sous forme de cépée saine en raison d'une part de sa localisation dans les versants difficilement accessibles et d'autre part épargnée suite à la présence du chêne vert qui constitue pour la population riveraine une excellente source de bois de chauffage. La connaissance de la dynamique des peuplements de frêne dimorphe constitue un élément indispensable à l'élaboration de scénarios et variantes de gestion raisonnée de ces écosystèmes preforestiers. 


\section{Structure diamétrique}

La structure diamétrique totale, ou répartition des tiges par classes de diamètre, est établie en prenant en compte tous les individus inventoriés (Roullet 1974; Gaudin 1996). Elle est porteuse d'information sur la stabilité (équilibre) du peuplement (Favrichon 1998). Pour des raisons pratiques nous avons choisi les classes suivantes:

$\varnothing<7,5 \mathrm{~cm}$ : Perches (P);

$7,5<\varnothing<22,5$ : Petit bois $(\mathrm{PB})$;

$22,5<\varnothing<42,5$ : Moyen bois (MB);

$42,5<\varnothing<62,5:$ Gros bois (GB);

> 62,5 cm: Très gros bois (TGB).

Les données recueillies de l'ensemble des relevés montrent que les classes les mieux représentées dans la frênaie étudiée sont respectivement les perches, avec un effectif de 226 individus, soit 42,40\% de l'effectif total, le petit bois avec un taux de 36,60\%, soit 195 individus et le moyen bois représenté par 112 individus, soit $21 \%$ de l'effectif global (Fig. 3). De manière générale, l'aspect de la structure définit la jeunesse des cépées qui forment le taillis, ou les classes de gros bois et très gros bois sont totalement absentes.

Le diamètre moyen de l'ensemble de la frênaie est de 13,29 cm. Cette valeur est nettement supérieure à celle estimée par Chafai (2016) qui lance une valeur moyenne de 5,17 $\mathrm{cm}$ pendant l'étude de la dynamique spatiale et de la biomasse du frêne dimorphe dans la cédraie de l'Ouled Yagoub (wilaya de Khenchela).

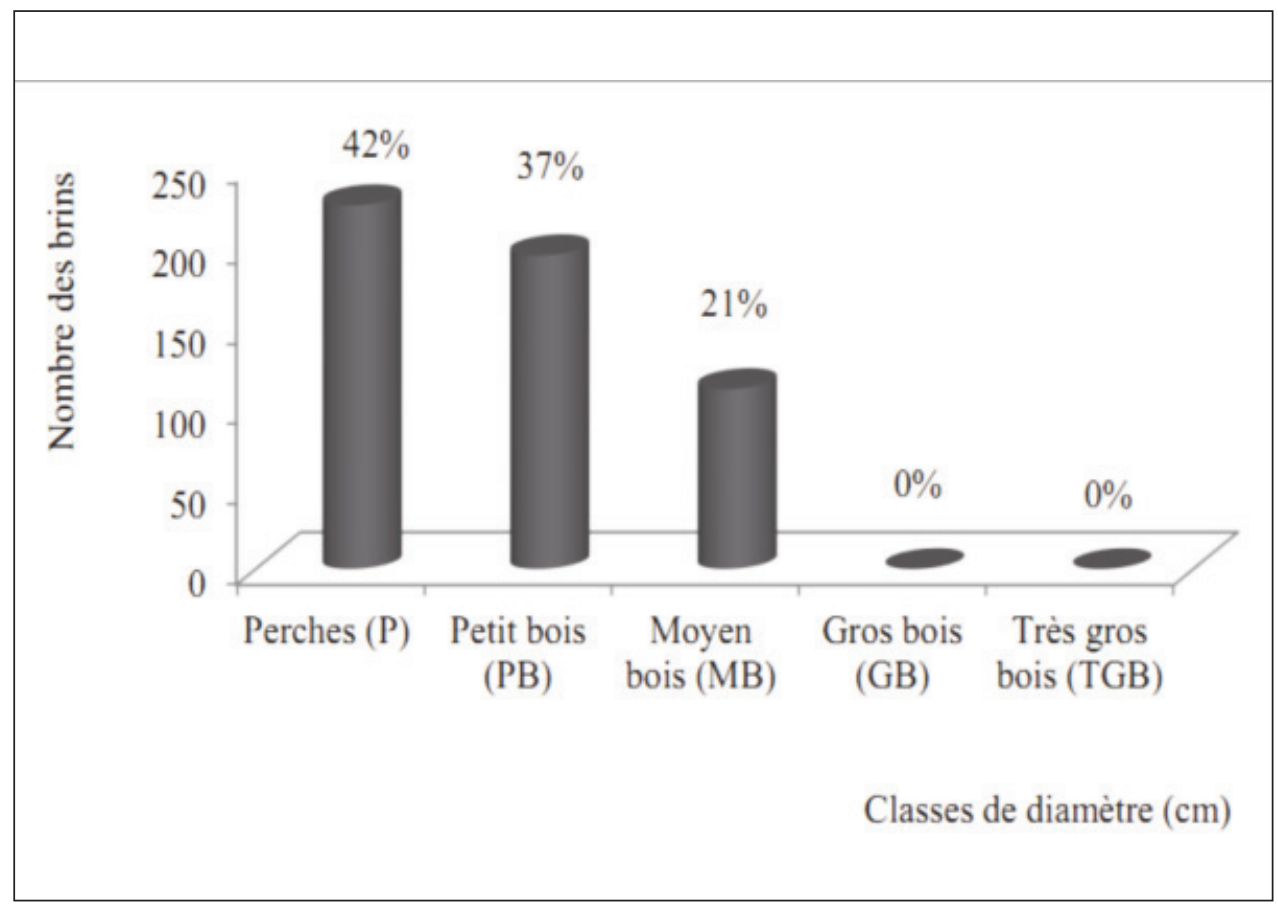

Fig. 3. Structure diamétrique du peuplement de frêne dimorphe dans les Monts des Ksour occidentaux. 


\section{Structure verticale}

La structure verticale représente la distribution des individus par classes de hauteur. Elle offre, entre autres, l'intérêt de pouvoir fournir un indicateur de richesse du site (Favrichon $\&$ al. 1998). Elle renseigne sur les potentialités de la station et sur le dynamisme du peuplement forestier (M'Hirit 1982).

Les hauteurs des sujets échantillonnés sont regroupées ci-dessus en classes pour facilité leurs représentation graphique.

Classe 1: 1 - 2,50 m;

Classe 2: $2,50-4 \mathrm{~m}$;

Classe 3: $4-5,50 \mathrm{~m}$;

Classe 4: $+5,50 \mathrm{~m}$.

La hauteur moyenne du peuplement varie de 1,80 à $6 \mathrm{~m}$. La valeur moyenne pour l'ensemble de la frênaie est $3,70 \mathrm{~m}$. La figure 4 schématise la distribution des classes de hauteur en fonction des effectifs. Elle révèle la dominance de la classe [2,50 - $4 \mathrm{~m}]$, suivie de celle de $[1-2,50 \mathrm{~m}]$, et en troisième place la classe $[4-5,50 \mathrm{~m}]$. Les fréquences respectives sont $43,71 \%, 27,95 \%$ et $21,95 \%$ soit un total de $93,62 \%$. Les sujets dont les hauteurs dépassent 5,50 m ne représentent que 6,38\%. Malheureusement la faible présence de cette classe rend cette structure sujette à des perturbations, de ce fait ce peuplement semble être déséquilibré.

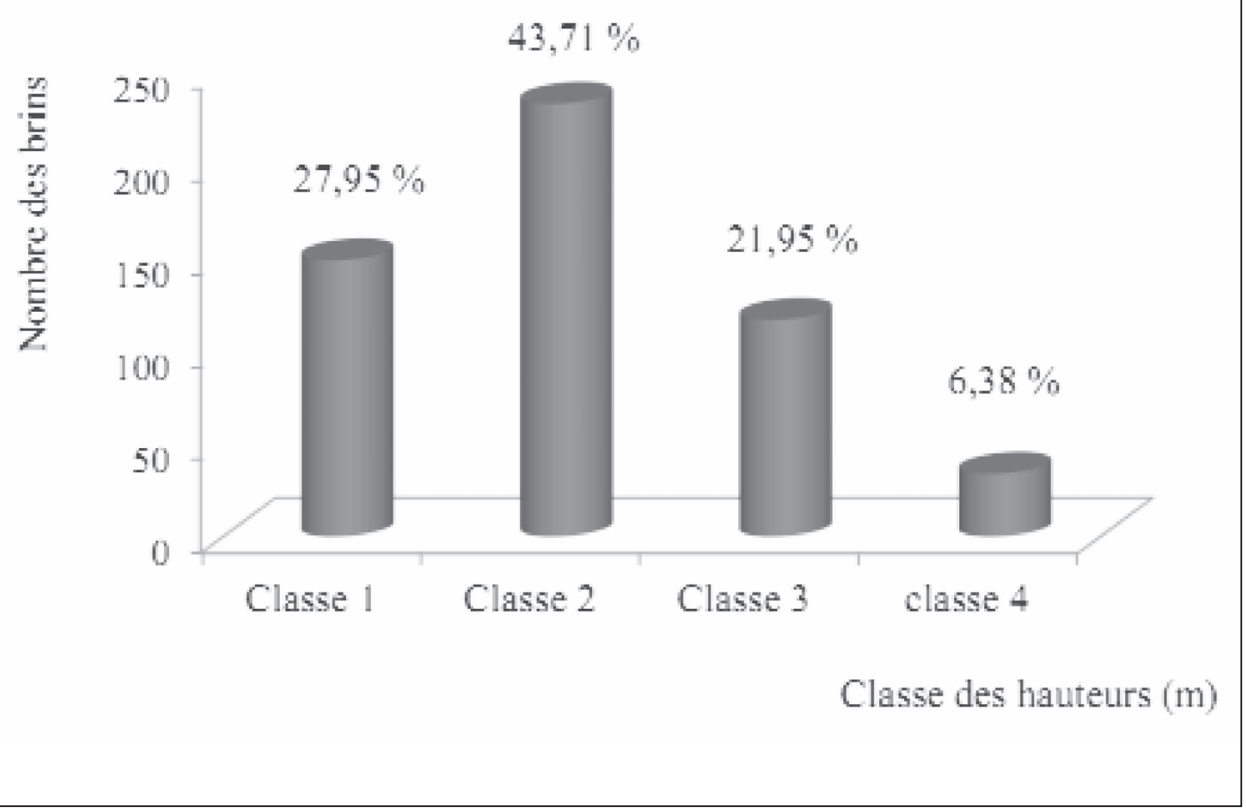

Fig.4. Structure verticale du peuplement de frêne dimorphe dans les Monts des Ksour occidentaux. 


\section{Conclusion et perspectives}

Les plantes rares, endémiques ou menacées de n'importe quel pays ou région sont de toute évidence celles qui suscitent le plus d'intérêt en matière de recherches, de protection et d'estimation de la biodiversité. En effet, en plus de leur intérêt biogéographique, elles sont rares dans un certain sens à l'échelle mondiale.

Fraxinus dimorpha, espèce menacée suivant la liste rouge de l'Union Internationale pour la Conservation de la Nature (UICN) dans la catégorie EN en danger, protégée par la loi algérienne selon le décret exécutif $n{ }^{\circ} 12-03$ du04 janvier 2012 établissant la liste des espèces incultes protégées, sa présence dans les Monts des Ksour témoigne donc de la particularité des conditions environnementales, difficilement réalisable dans les autres zones forestières algériennes. Pour préserver l'espèce et ses habitats natifs, les actions suivantes sont recommandées:

Cartographie de l'ensemble des frênaies des Monts des Ksour

Protection des sites potentiels contre la perte et la fragmentation de l'habitat, la déforestation, la collecte excessive et le surpâturage.

Mise en clôture des sites vulnérables pour protéger l'espèce de la surexploitation, du surpâturage, du piétinement et de la collecte impitoyable.

Création d'aires protégées pour assurer la régénération complète des espèces, des écosystèmes et pour restaurer la qualité des milieux sauvages.

Sensibilisation des usagers sur la gestion participative de ces territoires.

Estimation exacte de la taille des populations et étude de leurs dynamiques, tendances, biologie et écologie.

Identification des peuplements porte graines et création d'une banque de semences.

\section{Références bibliographiques}

Abdessemed, K. 1981: Le Cèdre de l'Atlas dans les massifs de l'Aurès et du Belezma, Étude phytosociologique et problèmes de conservation et d'aménagement. - Thèse deDoctrat. Fac. S.t. Jérôme. Université d'AixMarseille III.

Aidoud, A. 2005: Fonctionnement des écosystèmes méditerranéens. - Conférences, Université de Rennes.

Ait Ouali, R. 1991: Lerifting des Monts des Ksour au Lias, Organisation du bassin, diagénèse des assises carbonatées, Place dans les ouvertures mésozoïques du Maghreb, - Thèse de Doctorat en Sciences, Université d'Alger.

Amghar, F.\& Kadi Hanifi, H: 2008. Diagnostic de la diversité floristique de cinq stations steppiques du Sud Algerois. - Cahiers Orphée: 386-395.

Barbero, M. Loisel, R. Medail, F. \& Quézel, P. 2001: Signification biogéographique et biodiversité des forêts du bassin méditerranéen. - Bocconea 13: 11-25.

Battandier, J. A. \& Trabut L. C. 1895: Flore de l'Algérie. Monocotylédones, 2. - Paris.

Bellakhdar, J. 1997: La pharmacopée marocaine traditionnelle, Médecine arabe ancienne et savoirs populaires. - Paris.

Bonnet, V., Vidal, E. Medail, F. \& Tatoni, T. 1999: Analyse diachronique des changements floristiques sur un archipel méditerranéen périurbain (îles du Frioul, Marseille). - Ecol. Terre Vie 54: 3-18.

Boudy, P. 1950: Economie Forestière Nord-Africaine, Monographies et traitements des essences forestières, 2. - Paris. 
Chafai, C. 2016: Contribution à l'étude de la dynamique spatiale et de la biomasse du frêne dimorphe dans la cédraie de l'Ouled Yagoub Wilaya de Khenchela, - Thèse Magister, Univ Batna.

Charco, J. 2001: Guia de los arboles y arbustos del Norte de Africa: claves de determinacion, descripciones, ilustraciones y mapas de distribucion. - Madrid.

Daget, Ph. 1980: Sur les types biologiques botaniques en tant que stratégie adaptative. - Pp. 89-114 in: Barbault, R., Blandin, P. \& Meyer J. A. (eds) Recherches d'écologie théorique. - Paris.

Delassus, L. 2015: Guide de terrain pour la réalisation des relevés phytosociologiques, document technique. - Brest.

Dobignard, A. \& C. Chatelain, C. 2010-2013: Index synonymique flore d'Afrique du Nord, 1-5. Genève.

Emberger, L. 1938: Les Arbres du Maroc et comment les reconnaitre. - Paris.

Euro + Med. 2015. Euro+Med PlantBase. Berlin-Dahlem. - Available at: http://ww2.bgbm.org/EuroPlusMed/query.asp. [Dernier accès 13/03/2020]

Favrichon, V. Gourlet-Fleury, S. Bar-Hen, A. \& Dessard, H. 1998: Parcelles permanentes de recherché en forêt dense tropicale humide. Eléments pour une méthodologie d'analyse des données. Série FORAFRI. CIRAD-Forêt. - Montpellier.

Fennane, M. \& Ibn Tattou, M. 2005: Flore vasculaire du Maroc: inventaire et chorologie. - Rabat.

— \& - 2007: Flore pratique du Maroc, Manuel de détermination des plantes vasculaire, 2. - Rabat.

Fillali, K. 2011: Etude hydrogéologique du synclinal de Nâma (Monts des Ksour, Atlas saharien occidental). - Thèse Magister, Faculté des sciences de la terre et d'aménagement de territoire, université d'Oran.

Gaston, K. J. 1991: How large is species geographical range? - Oikos, 61: 434-438.

Gaudin, S. 1996: Dendrométrie des peuplements. BTSA Gestion forestière module D42. - Besançon.

Genin, D. Crochot, C. Msou, S.Araba, A. \& Alifriqui, M. 2016: Meadow up a tree: Feeding flocks with a native ash tree in the Moroccan mountains. - Pastoralism: Res. Policy Practice 11: 112. https://doi.org/10.1186/s13570-016-0058-9

Grime, J. P. 1977: Evidence for the existence of three primary strategies in plants and its relevance to ecological and evolutionary theory. - American Naturalist. 3: 1169 -1194.

Hamada, S. Dakki, M. Ibn Tattou, M.Ouyahya, A. etFennane, M. 2004: Analyse de la biodiversité floristique des zones humides du Maroc, Flore rare, menacée et halophile. - Acta Bot. Malacitana 29: 43-66. https://doi.org/10.24310/abm.v29i0.7226

Kacemi, A. Talbi, D. \& Ben Salah, M. 2011: Mise en évidence d'une déformation synsédimentaire transverse en compression au passage Lias Dogger (Secteur de Zerga - Monts des Ksour Atlas saharien - Algérie). - Geo-Eco-Trop 35: 81-90.

Kostova, I. etIossifova, T. 2007: Chemical components of Fraxinus species. - Fitoterapia 78: 85106. https://doi.org/10.1016/j.fitote.2006.08.002

M'sou, S. Genin, D. Romane, A. \& Alifriqui, M.2016: L'effet de la pratique de l'anastomose des tiges du Frêne dimorphe: Fraxinusdimorpha sur la production foliaire. - Pp. 24-29 in: 1st Mediterranean Plant Conservation Week. - Ulcinj.

Madon, O. \& F. Médail, F. 1996: The ecological significance of annuals on a Mediterranean grassland (Mt Ventoux, France). - Pl. Ecol. 129: 189-199

Maire, R. 1916: La végétationdes montagnes du sud oranais. - Alger.

-1952-1987: Flore de l'Afrique du Nord, 1-16. - Paris.

M'Hirit, O. 1982.: Etude écologique et forestière des cédraies du Rif Marocain, Essai sur une approche multidimensionnelle de la phytoécologie et de la productivité du cèdre (Cedrus atlantica Manetti), - Thèse de doctorat des sciences, université d'Aix - Marseille III.

Ozenda, P. 2004: Flore et végétation du Sahara, $3^{\circ}$ Ed. - Paris.

Paccalet, Y.1981: La flore méditerranéenne. - Paris.

Pignatti, S. 1982: Flora d'Italia, 1. -Bologna. 
Quézel, P. 1983: Flore et végétation actuelles de l'Afrique du Nord, leur signification en fonction de l'origine, de l'évolution et des migrations des flores et structures des végétations passée, Bothalia 1-14: 411-416. https://doi.org/10.4102/abc.v14i3/4.1186

— \& Santa, S. 1962-1963: Nouvelle flore de l'Algérie et des régions désertiques méridionales. Paris.

Rankou, H., M'Sou, S., Alifriqui, M. \& Martin, G. 2017: Fraxinus dimorpha. The IUCN Red List of Threatened Species 2017: e.T109366166A109366170. https://dx.doi.org/10.2305/ IUCN.UK.2017-3.RLTS.T109366166A109366170.en [Last Accessed 21 March 2020]

Raunkiaer, C. 1934: The life form of plants and statistical plant geography. - Oxford.

Roullet, B. 1974: Etudes quantitatives d'une forêt dense humide sempervirente de la Guyane vénézuélienne. - Thèse de Doctorat, Fac. Sc. Université deToulouse.

Taleb, M. S. \& Fennane, M. 2008: Diversité floristique du parc national du Haut Atlas Oriental et des massifs Ayachi et Maâsker (Maroc). - Acta Bot. Malacitana 33: 125-145. https://doi.org/10.24310/abm.v33i0.6979

Yelles Chaouche, A. K. Ait Ouali, R., Barcene, R., Derdere, M. E. M. \& Djelit, H. 2001: Chronologie de l'ouverture du bassin de Ksour (Atlas saharien, Algérie) au début du mesozoique. - Bull. Soc. Géol. France 172(3): 285-293. https://doi.org/10.2113/172.3.285

Adresses des auteurs:

Kouider Hadjadj ${ }^{1 *}$, Mohammed Benaissa ${ }^{1}$, Mohammed Mahammedi ${ }^{1}$, Gordo Belkacem², Lakhdar Guerine ${ }^{3}$,

${ }^{1}$ Faculté des sciences de la nature et de la vie, Université de Djelfa, Algérie. Email: hadjadjkouider@gmail.com

${ }^{2}$ Département des ressources forestières, Facultés des sciences de la nature et de la vie et des sciences de la terre et de l'univers, Université de Tlemcen, Algérie.

${ }^{3}$ Département de physiologie végétale, Faculté des sciences de la nature et de la vie, Université d'Oran, Algérie.

${ }^{4}$ Faculté des sciences de la nature et de la vie, Centre universitaire de Naâma, Algérie.

* Auteur correspondant 
